\title{
Search for annihilating Dark Matter towards dwarf galaxies with the Cherenkov Telescope Array
}

\author{
Aldo Morselli ${ }^{1}$ and Gonzalo Rodríguez ${ }^{1, a}$ for the CTA Consortium ${ }^{2}$ \\ ${ }^{1}$ Sezione INFN di Tor Vergata, Roma, Italy \\ ${ }^{2}$ See www.cta-observatory.org for full author and affiliation list
}

\begin{abstract}
The standard model of cosmology indicates that approximately 27\% of the energy density of the Universe is in the form of dark matter. The nature of dark matter is an open question in modern physics. Indirect dark matter searches with imaging atmospheric Cherenkov telescopes are playing a crucial role in constraining the nature of the dark matter particle through the study of their potential annihilation that could produce very high energy gamma rays from different astrophysical structures. The Cherenkov Telescope Array will provide an unprecedented sensitivity over a range of dark matter mass from $\sim 100 \mathrm{GeV}$ to $\sim 30 \mathrm{TeV}$. In this contribution we review the status of indirect dark matter searches at dwarf spheroidal galaxies.
\end{abstract}

\section{Introduction}

The existence of dark matter (DM) in our Universe (27\% of the total energy) is well established, but its nature is still unknown. Among the most promising particle candidates are Weakly Interacting Massive Particles (WIMPs), which typically can self-annihilate and generate gamma rays $[1,2]$. If WIMPs particles are produces thermally in the early Universe then the self-annihilation cross-section has a natural value of approximately $3 \times 10^{-26} \mathrm{~cm}^{3} \mathrm{~s}^{-1}$ [3]. WIMPs models, such as the supersymmetric neutralino give prediction the for gamma-ray energy spectra from the annihilations, which are crucial inputs, together with the DM distribution in the observed target, to achieve prospects for the sensitivity of indirect searches [4]. The goal of the present study is to provide preliminary comparative expectations on indirect DM searches with the Cherenkov Telescope Array [5], taking into account continuum gamma-ray signatures coming from typical DM annihilation channels.

\section{Cherenkov Telescope Array performance}

The Cherenkov Telescope Array (CTA) is a next-generation imaging atmospheric Cherenkov telescopes (IACTs) which is currently in the planning and prototyping stage and scheduled to finish construction in 2020. In order to provide full sky coverage, the CTA observatory will consist of two arrays, one in the southern hemisphere close to Paranal Observatory (Chile), and a second one in the northern hemisphere in the Observatorio del Roque de Los Muchachos on the island of La Palma

a e-mail: grferna@roma2.infn.it 
(Spain). An improved sensitivity over the full energy range as well as improved energy and angular resolution with respect to current generation IACTs, will be achieved by combining many telescopes of three different sizes distributed over a large area: few large size telescopes (LST), several medium size telescopes (MST), and many small size telescopes (SST). The baseline northern array will consist of 4 LSTs and 15 MSTs and the southern one will be composed of 4 LSTs, 25 MSTs and 70 SSTs. An extension for the southern observatory is envisaged based on a fourth, medium-sized Schwarzschild Couder telescope type. The expected performance of CTA has been evaluated with very detailed Monte Carlo simulations for many different layouts and sites [6]. The expected angular resolution of CTA is compared with that of some current and future gamma-ray detectors in the left panel of figure 1. The angular resolution of CTA will be better than that of any current IACT array over the full energy range. The CTA differential flux sensitivity for both southern and northern arrays for an observation time of 50 hours is compared in the right panel of figure 1 with the sensitivities of MAGIC-II and VERITAS for the same observation time. HAWC sensitivities for an observation time of one year and five years are shown too for comparison. The flux sensitivity of the CTA northern array will be less than that of the southern one over the entire energy range due to the lower number of telescopes and different site altitude.
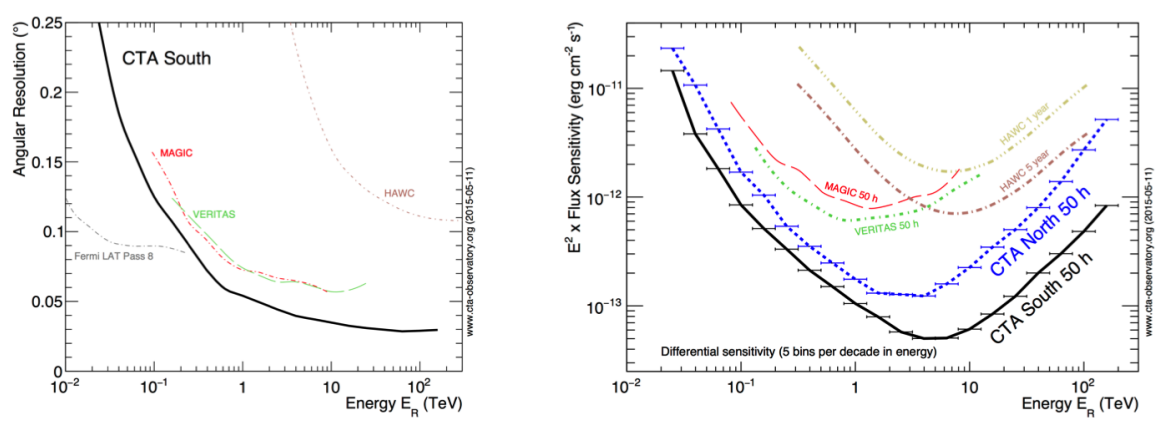

Figure 1: Left panel: Angular resolution (68\% containment radius of the gamma-ray PSF) as a function of energy for CTA southern array (black line). Right Panel: Expected differential sensitivity for CTA southern array (black line) and CTA northern array (blue line). Figure from [7]

\section{Indirect Dark Matter searches}

The indirect detection experiments aim at searching for a flux of annihilation products created in astrophysical environments where DM annihilation may be occurring at an appreciable rate [8]. In particular, the indirect DM searches carried out by IACTs look for photons from WIMPs in the mass range $\sim 100 \mathrm{GeV}$ up to $\sim 100 \mathrm{TeV}$. The flux expected from a DM-dominated region depends respectively on the so-called particle physics and astrophysical (or $J$ ) factors:

$$
\Phi_{s}(\Delta \Omega)=\frac{1}{4 \pi} \frac{<\sigma v>}{2 m_{D M}^{2}} \int_{E_{\min }}^{E_{\max }} \frac{d N_{\gamma}}{d E_{\gamma}} d E_{\gamma} \times J(\Delta \Omega),
$$

where $\langle\sigma v\rangle$ is thermally averaged self-annihilation cross-section times velocity, $m_{D M}$ is the dark matter particle mass, $E_{\min }$ and $E_{\max }$ are the energy limits for the measurement and $\frac{d N_{\gamma}}{d E_{\gamma}}$ is the energy spectrum of the gammas produced in the annihilation (as, e.g., from [9]). The J-factor is the integral 
along the line of sight of the squared DM density profile of the given target integrated within an aperture angle, $\int_{\Delta \Omega} \mathrm{d} \Omega \int_{l \text { lo.s. }} \rho_{D M}^{2}(\mathbf{r}) \mathrm{d} l \mathrm{~d} \Omega^{\prime}$. The products of DM annihilation are thought to come from decay and/or hadronization of the primary Standard Model (SM) particles: quark-antiquark, lepton and boson, and each channel is expected to have its own branching ratio.

\section{Dwarf Spheroidal Galaxies}

The indirect dark matter search with CTA has several possible astrophysical targets each with its own inherent advantages and disadvantages. The Galactic Center (GC) and the Dwarf Spheroidal Galaxies (dSphs) are the most promising targets. The large amount of DM and its proximity makes the GC a very interesting target to search for DM annihilation signals. However, the expected high background in very high energy gamma rays makes it hard to separate the DM signal from the gammarays of astrophysical origin. On the other hand, the $\mathrm{dSphs}$ are believed to be the smallest and faintest astronomical objects whose dynamics is dominated by DM, and they are free from gamma-ray astrophysical backgrounds [10]. Furthermore, ongoing optical surveys (such as DES, PanSTARSS, VLT, ATLAS), devoted to explore large areas of the sky, are increasing the pool of new dSphs in the Local Group and the knowledge needed to better constrain the values of the $J$-factors. On the basis of the Dark Energy Survey (DES) two years data [8], some of the newly discovered satellites in the southern hemisphere could already be considered among the most promising targets to be observed for the first time by the next generation of IACTs. The final choice of the most promising dwarf galaxy targets for CTA observations will be made at the start of array operation based on all available information at that time. In the left panel of figure 2 we show the sensitivity for different annihilation channels for the classical dSphs Sculptor [11]. On the right panel of the same figure we compare the sensitivity for Sculptor, Draco, Coma Berenices and Segue 1, showing the $\pm 1 \sigma$ limits on the $J$-factor uncertainty.
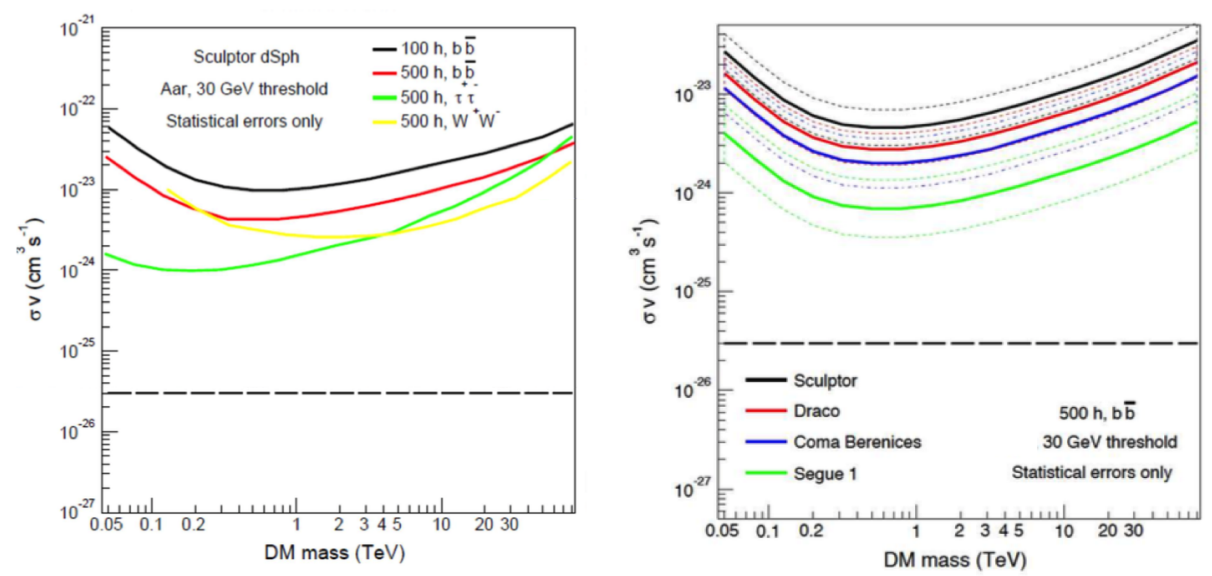

Figure 2: Left panel: Sensitivity for $\sigma v$ from observation of the classical dwarf galaxy Sculptor for different annihilation modes as indicated. Right panel: Sensitivity for $500 \mathrm{~h}$ observation of the classical dSphs Draco and Sculptor, and the ultra-faint dwarf galaxies Segue 1 and Coma Berenices as indicated. Dashed lines correspond to $\pm 1 \sigma$ on the $J$-factors. Sensitivity is computed assuming the $b \bar{b}$ annihilation channel. 


\section{Conclusions}

The Cherenkov Telescope Array will improve the sensitivity to DM annihilation for a range of interesting DM masses. The expected improvement in the angular resolution means that some dwarf spheroidal galaxies could be resolvable; the typical point-source assumption will no longer be valid, and the density profile could be constrained using gamma-ray measurements. Observations of dwarf spheroidal galaxies with CTA will be valuable for providing robust legacy constraints (in case of no detection). There is also a good complementary in the WIMPs mass range with other experiments like Fermi, MAGIC [12] and ANTARES [13]. New ultra-faint dwarf spheroidal galaxies are being and will be discovered with the next generation of sky surveys. When CTA starts to operate the best constrained/most promising known dwarf spheroidal galaxies will be chosen for observation time.

\section{References}

[1] L. Bergstrom, Non-Baryonic Dark Matter - Observational Evidence and Detection Method, Rep. Prog. Phys. 63, 793 (2000).

[2] G. Bertone, D. Hooper and J .Silk, Particle Dark Matter: Evidence, Candidates and Constraints, Phys. Rep. 405, 279 (2005).

[3] Steigman G. et al, Precise relic WIMP abundance and its impact on searches for dark matter annihilation, Phys. Rev. D 86, 2, 023506 (2012).

[4] S. P. Martin, A Supersymmetry Primer, arXiv:hep-ph/970935.

[5] Acharya B. S. et al, (CTA Consortium), Introducing the CTA concept, Astro. Phys. 43, 3 (2013).

[6] K.Bernlohr et al.(CTAConsortium),Monte Carlo design studies for the Cherenkov Telescope Array, Astroparticle Physics 43 (2015) 171-188.

[7] CTA Construction Project Science Technical Design Report, OBS-TDR/141106

[8] M. Cirelli et al, PPPC 4 DM ID: A Poor Particle Physicist Cookbook for Dark Matter Indirect Detection, , JCAP 1103 (2011) 051, erratum: JCAP 1210 (2012) E01, arXiv: 1012.4515, http://www.marcocirelli.net/PPPC4DMID.html.

[9] Drlica-Wagner A. et al. (2015), arXiv:1508.03622

[10] Bergstrom L. (2012), Annalen der Physik, vol 524 issue 9-10, pp479-496

[11] Carr et al. arXiv:1508.06128

[12] M. L. Ahnen et al. arXiv:1601.06590

[13] A. Albert et al. arXiv:1612.04595 